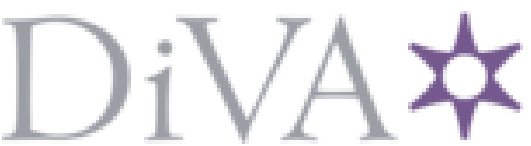

http://www.diva-portal.org

Postprint

This is the accepted version of a paper presented at IEEE 11th International Conference on Mechanical and Intelligent Manufacturing Technologies.

Citation for the original published paper:

Rahimi, F. (2020)

Concurrent and Optimal Structure, Control and Implementation Design In:

N.B. When citing this work, cite the original published paper.

Permanent link to this version:

http://urn.kb.se/resolve?urn=urn:nbn:se:kth:diva-276826 


\section{Concurrent and Optimal Structure, Control and Implementation Design}

\author{
Fariba Rahimi \\ Dept. Machine Design \\ KTH Royal Institute of Technology \\ Stockholm, Sweden \\ email: frahimi@kth.se
}

\author{
Jan Wikander \\ Dept. Machine Design \\ KTH Royal Institute of Technology \\ Stockholm, Sweden \\ email: Janwi@kth.se
}

\begin{abstract}
Mechatronic system design includes a combination of different engineering disciplines. A common approach in design of mechatronic systems is based on a sequential method, where different disciplines are treated and designed separately. This paper extends earlier work on integrated physical and control design optimization with integrating an additional aspect of the corresponding embedded control system implementation. Our previous publications describe integrated design optimization through a few specific use cases but the impact of embedded control implementation on the structural design of the systems is neglected. In this paper, the approach is extended to cover discussions on control implementation and its effect on the physical dimensioning and vice versa. A multi-objective optimization approach is implemented and tested on a mechatronic system case study consisting of a DC-motor, a planetary gear, a flexible shaft, an embedded controller and a load. The couplings between the properties of different engineering domains are studied and highlighted. The presented approach which is aimed for early phases of design, considers the integration of three engineering disciplines in one design framework which so far has been missing.
\end{abstract}

Index Terms - component, physical design, control implementation, static transformation, dynamic behavior model

\section{INTRODUCTION}

Simultaneous design of mechanics and electronics, hardware and software and embedded control functions resulting in an integrated component or system are all of significant importance [1]. Designing embedded control systems has been considered in two dependent steps, first the controllers are synthesized and, second these controllers are mapped and scheduled on a given platform. This approach often leads to either resource underutilization or poor control performance and maybe even lead to instability of control applications, because of the timing problems that arise due to certain implementation decisions [2], [3].

A common simplification adopted in previous studies in the design optimization of mechatronic systems is to ignore the influence of embedded control implementation on the final control performance. As is well known, decisions on embedded implementation, e.g., the sampling period, real-time scheduling methods, and communication delays, may have a large impact on the performance of the final systems. Improper selection of these implementation related parameters may significantly deteriorate the control performance or even cause the system to be unstable [4]. There are many research contributions on the integration of mechanism and control design, as well as studies on the co-design of control and embedded implementation; however, there is no integrated design method that considers the design of mechanism, control algorithm, and embedded implementation all together. An optimization method capable of integrating physical and control design, together with controller implementation aspects is likely to lead to better engineering results. Thus, to address the aforementioned gaps and problems, the overall design optimization problem should include the indispensable embedded control implementation influence.

Previously published work by the authors [5], [6], [7], [8] presented a software framework called IDIOM (Integrated Design and Optimization of Mechatronic systems) where the main focus was on optimizing the system for one primary objective such as the size or weight of the system without taking into account the control implementation impact on the design and vice versa.

The component library of the presented IDIOM framework consists of two kinds of component models identified as physical and control components. Each physical component includes three main models, namely physical dimensioning model, static force/torque transformation model and dynamic behavior model. Each control component carries information about the control method and control performance constraints such as overshoot, rise time and ISE (integrated square error). The targeted mechatronic system is configured using these component models. The overall requirement on the system to be designed is formulated as a load inertia and motion profile. All physical dimension constraints and control performance constraints should be satisfied for the given load profile. The method has so far ignored control implementation effects on the physical and control design optimization, and hence continuous time modeling has been sufficient.

In this paper, the method is improved to facilitate inclusion of control implementation aspects such as sampling rate, delays and sensor resolution. To evaluate the feasibility of the extended method, it is implemented on a design case consisting of a dc-motor, a gear stage, a flexible shaft and a load.

The outline of the paper is as follows, Section I is an introduction to the presented approach and includes a few 
motivational references and a short summary of previous work. Section II includes a short literature review of related research. Section III presents the design methodology, the physical modelling and control method together with the implemented optimization approach, the objectives of the design and introduced detailed constraints. Section IV introduces the case study with its requirements. Section V presents the achieved results and includes a discussion. The paper is concluded in Section VII.

\section{STATE OF THE ART}

The developments for mechatronic systems, design approaches and examples of mechatronic products considering various embedded control functions and system's integrity are summarized in [1]. Broenink et al. [9] presented a method to support design and implementation of control software for mechatronic systems. They consider the process as a stepwise method from physical system models and control laws to efficient computer code.

A holistic co-design approach for robot arm structure is presented in [10]. Their approach includes optimization of the design parameters describing arm link geometry, actuator locations and feedback gains considering the settling time of the system as objective function. The structural parameters are optimized using a non-linear programming technique to obtain an overall optimal performance.

A method that combines experimentation with a design process is developed in [11] to integrate design and control of mechatronic system. The control gains are optimally tuned. Using those optimal values, the mechanical structure is modified until the control performance is improved. An optimal design of mechatronic systems with configurationdependent dynamics is considered in [12]. They have tested their approach on a pick and place robot with a gripper and studied the non-convex nature of the optimization problem and challenges in modeling of a serial machine with flexible components and its embedded controller.

An approach for designing an optimal control for "hybrid' mechanical systems is presented and implemented on a compass gait biped in [13]. They refer to both continuous dynamics that are in the form of Lagrangian equations and discrete behaviour of different motion phases of the biped as 'hybrid'. They have used Discrete Mechanics and Optimal Control (DMOC) to formulate optimal control as a constrained optimization problem. Formulation and solution of a control and scheduling co-design problem is presented in [14]. They have optimized overall performance of control loops for a given set of plants with disturbances and measurement noise.

Simon et al. [15] proposed a method for robust control and scheduling co-design. They tuned the control periods according to their impact on the control performance and then regulated an outer scheduling controller in real-time according to the allocated computing power. Subsequently, Simon et al. [16] presented a methodology to design a robust controller by taking the sampling interval into account as a control variable of the system. They have considered plant parameter uncertainties in robustness of the control. Cervin and Eker [17] presented design of a flexible real-time control system for implementation of feedback control tasks in embedded systems. They have studied some features of the model from control implementation perspective such as latency and sampling time/ jitter.

Non of the above papers considers the effect of control system implementation on the physical system design. In this paper, the aim is to find a balanced optimum between the physical structure (including transmissions and actuator), the control design and embedded control implementation by enabling integration of the three engineering domains already in an early design phase. This should avoid cost and time consuming design iterations in later design stages. The approach is focused on dimensioning of the physical system in order to get an optimum (small in size) design together with considering the control implementation (optimum sampling rate).

\section{Physical Design and Control Optimization of A MEChatronic System}

Our approach to system optimization would be classified as a simultaneous approach [18] where the optimized system is acquired by concurrently varying both the plant and controller parameters. Including the effect of control implementation in the design optimization requires that previous work is extended to include also discrete time models of the closed loop system. In this paper, a key design parameter for an embedded controller implementation, namely the sampling time is added as an additional free variable in the design optimization.

The methodology for the integrated physical design and control optimization is given in Fig. 1 where the static transformation models are executed throughout the chain of physical component models using the load profile to determine the required torque to be handled by each component. Based on the required load profile for each component, the physical dimensioning models are executed and the satisfaction of physical constraints are checked to ensure that non-feasible component dimensions are rejected. Subsequently, the dynamic behavior model of components together with interface equations are configured to form the open loop symbolic transfer function or state space model. Adding the control method, the symbolic closed loop system is modeled.

The numerical closed loop system model is derived by substituting the symbolic entities with parameter values which satisfied the physical constraints. A nominal sampling period is calculated using the fastest pole of the closed loop system and a design parameter is defined as a coefficient for variation of sampling frequency. The output of the controlled system is derived and the satisfaction of physical constraints are evaluated against the actual force/torque on each component.

In this paper, the system is optimized for two main objectives, volume of the entire system and the sampling frequency. In ongoing work also additional embedded implementation aspects such as sensor resolution and control delays are investigated. This whole approach is applied for each optimization 


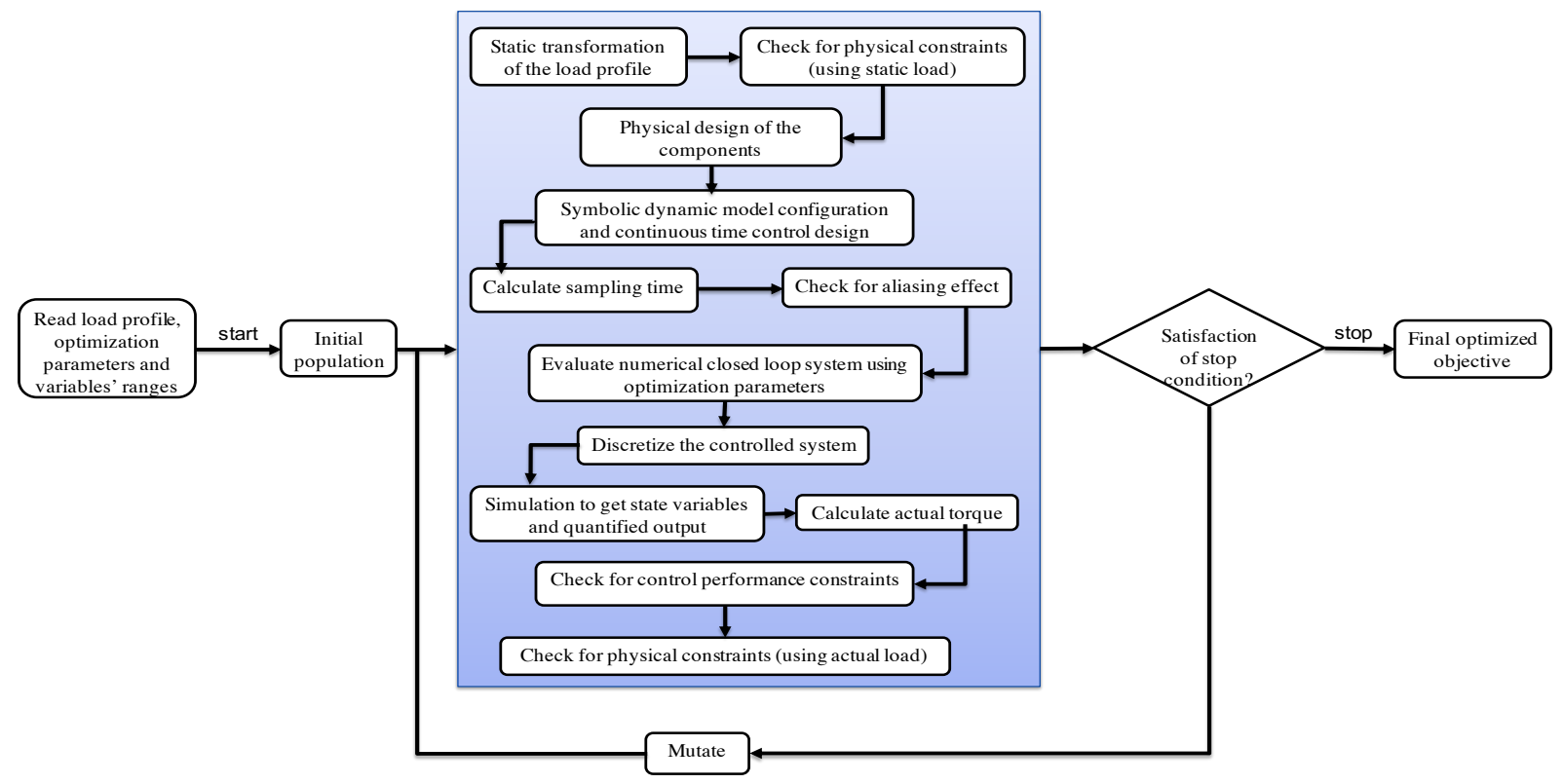

Fig. 1. Overview of the methodology

instant until the stop condition for the optimizer is satisfied. The final optimum solution is derived in terms of a pareto optimal front and later using a weighted sum method.

\section{A. Physical Model}

The method is based on two types of models, static and dynamic models. The static models describe the relations between design parameters of physical components. The dynamic models refer to the component behavior as functions of time. Both model types are necessary for the design and control performance evaluation and optimization.

The open loop system model for a configured system is a continuous time model as given in (1):

$$
\begin{aligned}
\dot{\mathbf{x}}(t) & =A \mathbf{x}(t)+B \mathbf{u}(t) \\
\mathbf{y}(t) & =C \mathbf{x}(t)+D \mathbf{u}(t)
\end{aligned}
$$

where $\mathbf{x}$ and $\mathbf{u}$ are the system states and control signal, respectively. The output of the system is denoted by $\mathbf{y}$.

So far, several different component models have been continuously developed and modeled. The models described in here are relevant to the case study that will be discussed in the next section. Dynamics of the physical component models together with the interface equations given in (2)-(14) are configured to formulate the continuous time state-space model.

\section{1) Dc-motor dynamics:}

$$
K_{T} i_{\text {in }}=T_{m, \text { out }}+J_{m} \ddot{\phi}_{m}
$$

where $K_{T}$ is the motor torque constant and $i_{i n}$ is the current. $J_{m}$ is the motor inertia and $T_{m, o u t}$ is the output torque.

\section{2) Planetary gear dynamics:}

$$
\left(T_{p g, \text { in }}-J_{p g} \ddot{\phi}_{p g, \text { out }}\right) n=T_{p g, \text { out }}
$$

$$
\phi_{p g, i n}=n \phi_{p g, o u t}
$$

where $T_{p g, i n}$ and $T_{p g, o u t}$ are the input and output torques of the gearbox. $J_{p g}$ and $n$ are the inertia and gear ratio, respectively.

3) Flexible shaft dynamics: The shaft is modeled as a lumped mass-spring-damper.

$$
\begin{gathered}
T_{s h, \text { in }}=k_{s h}\left(\phi_{s h, \text { in }}-\phi_{\text {sh }, \text { out }}\right)- \\
d_{s h}\left(\dot{\phi}_{s h, \text { in }}-\dot{\phi}_{s h, \text { out }}\right) \\
J_{\text {sh }} \ddot{\phi}_{s h, \text { out }}=k_{s h}\left(\phi_{s h, \text { in }}-\phi_{\text {sh }, \text { out }}\right)+ \\
d_{s h}\left(\dot{\phi}_{s h, \text { in }}-\dot{\phi}_{\text {sh,out }}\right)-T_{\text {sh,out }}
\end{gathered}
$$

$J_{s h}, k_{s h}$ and $d_{s h}$ are the inertia, stiffness and damping factors of the shaft.

4) Load dynamics:

$$
\begin{gathered}
J_{l} \ddot{\phi}_{l, \text { in }}=T_{l} \\
\phi_{l, \text { in }}=\phi_{l, \text { out }}
\end{gathered}
$$

where $J_{l}$ is the load inertia, $\phi_{l, \text { in }}$ and $\phi_{l, o u t}$ are the input and output load position profiles and $T_{l}$ is the load torque.

The interface equations between dynamics of each component are as following,

$$
T_{m, \text { out }}=T_{p g, \text { in }}
$$




$$
\begin{gathered}
\ddot{\phi}_{m, \text { out }}=\ddot{\phi}_{p g, \text { in }} \\
T_{p g, \text { out }}=T_{s h, \text { in }} \\
\ddot{\phi}_{s h, \text { in }}=\ddot{\phi}_{p g, \text { out }} \\
T_{\text {sh,out }}=T_{l} \\
\ddot{\phi}_{s h, \text { out }}=\ddot{\phi}_{l, \text { in }}
\end{gathered}
$$

Using the above dynamics of component models, and by defining the position of the output shaft $\left(\phi_{\text {sh,out }}\right)$ as the output of the system and $i_{i n}$ as the input and position and velocity of the input and output shaft as state variables, the symbolic open loop state space matrices, $A, B, C$ and $D$ are derived as follows,

$$
\begin{gathered}
A=\left[\begin{array}{cccc}
0 & 0 & 1 & 0 \\
0 & 0 & 0 & 1 \\
-\frac{k_{s h}}{n^{2} J_{\text {tot }}} & \frac{k_{\text {sh }}}{n J_{\text {tot }}} & -\frac{d_{\text {sh }}}{n^{2} J_{\text {tot }}} & \frac{d_{s h}}{n J_{\text {tot }}} \\
\frac{k_{s h}}{n J_{\text {out }}} & -\frac{k_{\text {sh }}}{J_{\text {out }}} & \frac{d_{\text {sh }}}{n J_{\text {out }}} & -\frac{d_{\text {sh }}}{J_{\text {out }}}
\end{array}\right] \\
B=\left[\begin{array}{c}
0 \\
0 \\
\frac{K_{T}}{J_{\text {tot }}} \\
0
\end{array}\right], C=\left[\begin{array}{llll}
0 & 1 & 0 & 0
\end{array}\right], D=0
\end{gathered}
$$

where $J_{\text {tot }}$ and $J_{\text {out }}$ are the total inertia on the motor side and load side as,

$$
\begin{aligned}
& J_{t o t}=J_{m}+J_{p g} \\
& J_{\text {out }}=J_{\text {sh }}+J_{l}
\end{aligned}
$$

\section{B. Control}

An example of a control component is a PD control (Fig. 2) with a low pass filter to overcome the impact of noise in the system. In the remaining part of this paper noise is however not considered.

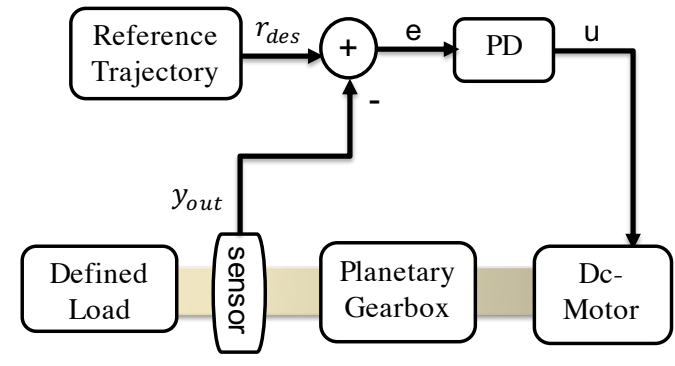

Fig. 2. Error feedback PD control

For the case of a PD controller, which is also used in the case study (Sec. IV), the continuous time controlled system is derived using (16) with control method in (15). The input vector, $\mathbf{r}_{\text {des }}(t)$, collects the reference input which is the load profile and the output signal, $\mathbf{y}_{\text {out }}(t)$, is defined to be the output angular position of the shaft. The control parameters $k p, k_{d}$ and $r_{0}$ are updated in between optimization.

$$
\begin{gathered}
G_{c}=k_{p}+\frac{k_{d} s}{s+r_{0}} \\
G_{c l}=\frac{G_{p} G_{c}}{1+G_{p} G_{c}}
\end{gathered}
$$

$G_{p}$ is equivalent to the linear model $A, B, C, D$.

The continuous time closed loop system and the required load profile are sampled and discretized. The discretized system is simulated in each optimization run according to Fig. 1. A good choice of sampling time is important for the approach. Clearly, a shorter sampling time typically gives the advantage of better performance (closer to the corresponding continuous time system). On the other hand, a longer sampling time gives the advantages of lower computational load and may reduce numerical challenges.

To address this trade-off issue the sampling frequency is defined as one of the optimization objectives. The sampling time is derived using (17),

$$
T_{s}=\frac{2 \pi}{c_{b} w_{b}}
$$

where $w_{b}$ is the fastest frequency of the closed loop system and $c_{b}$ is a frequency coefficient that is defined to be a real valued optimization parameter in the range of $[2 \ldots 30]$.

\section{Optimization}

\section{1) Optimization objective:}

A multi-objective optimization is considered to minimize volume of the mechatronic system as a physical design objective together with minimizing the sampling frequency as a control implementation objective.

$$
\min _{\hat{\mathbf{v}} \in \gamma} \sum_{i=1}^{n=2} o b j_{i}(\hat{\mathbf{v}})
$$

where $o b j$ is the objective of the optimization and $i=1, \ldots, n$ is the number of the objectives, $\hat{\mathbf{v}}$ is the physical and control optimization variables and $\gamma$ is the feasible solution set. Feasible solutions are those for which all optimization constraints are met.

Considering the non-linear nature of discrete time systems and including control performance evaluation require a capable numerical optimization method. To solve this multi-objective and computationally demanding optimization problem, a $\mathrm{Ge}$ netic Algorithm is used. As an example, which is also used in the case study (Sec. IV) we have,

$$
o b j_{1}=\min _{\left(l_{m}, r_{g}, l_{s h}\right) \in\left(\gamma_{1}, \gamma_{2}, \gamma_{3}\right)} v_{m}+v_{g}+v_{s h}
$$

where $v_{m}, v_{g}$ and $v_{s h}$ are the volume of the dc-motor, planetary gear and the shaft that sum up for the volume of the entire system as presented in (20)

$$
\begin{gathered}
v=\pi r_{\mathrm{m}}^{2} l_{\mathrm{m}}+ \\
\pi r_{\mathrm{g}}^{2}\left(b_{g}+b_{c}\right)+\pi r_{s h}^{2} l_{s h}
\end{gathered}
$$


where $b_{c}$ and $b_{g}$ are the carrier width of the gear and the total width of the gear, respectively. $r_{m}, l_{m}, r_{g}, r_{s h}, l_{s h}$ are the motor radius and length, gear radius and shaft radius and length, respectively.

The second objective function is the sampling frequency of the system given in (21) and is derived as in (22)

$$
\begin{gathered}
o b j_{2}=\min _{\left(c_{b}, k_{p}, k_{d}, r_{0}\right) \in\left(\gamma_{1}, \gamma_{2}, \gamma_{3}, \gamma_{4}, \gamma_{5}\right)} w_{s} \\
w_{s}=\frac{2 \pi}{T_{s}}
\end{gathered}
$$

where $T_{s}$ is variant according to (17).

The final optimized system is determined by weighted sum function using the pareto optimal front set,

$$
o b j=k_{1} v+k_{2} w_{s}
$$

where $k_{1}$ and $k_{2}$ are the pareto front weight factors decided based on the importance of the objectives. We have considered different values of $k_{1}$ and $k_{2}$ to evaluate the final optimum system and the impact of these parameters on each objective.

\section{2) Physical constraints of the optimization:}

To implement the physical constraints on the system, it is necessary to execute the static transformation of the load profile throughout the component models. This is done in two steps for each individual, first for the continuous time model and secondly during discrete time simulation with actual dynamic forces/torques.

The physical constraints for the DC-motor is adopted from scaling approaches presented in [19] and [20] which specify the relationship between the rated torque, $T_{m \text {,rated }}$ (based on manufacturer data), and the actual root mean square torque, $T_{r m s}$, as in (24). The rated torque is derived based on the mechanical, magnetic and thermal effects.

$$
\begin{gathered}
T_{m, \text { rated }} \geq T_{r m s} \\
T_{m, \text { rated }}=C_{m} l_{m} r_{m}^{2.5}
\end{gathered}
$$

$C_{m}$ is the motor type constant for the same cooling conditions, $l_{m}$ is the motor's rotor length and $r_{m}$ is the radius of stator. The motor's RMS torque is derived as,

$$
T_{r m s}=\sqrt{\frac{1}{\tau} \int_{0}^{\tau}\left(\left(C_{m j} l_{m} r_{m}^{4}+J_{m, s}\right) \ddot{\phi}_{m}+T_{m, \text { out }}\right)^{2} d t}
$$

$C_{m j}$ is a constant for specific machine type and is derived from a reference motor of the same type, $\tau$ is the cycle time of the output profile, i.e., the time period during which the output profile is valid, $\ddot{\phi}_{m}$ is the angular acceleration of the motor output. $T_{m, \text { out }}$ is the output torque of the motor and $J_{m, s}$ is the inertia of motor's shaft and bearings. Combining (24)-(26) results in (27).

$$
\begin{gathered}
C_{m} l_{m} r_{m}^{2.5} \geq \\
\sqrt{\frac{1}{\tau} \int_{0}^{\tau}\left(\left(C_{m j} l_{m} r_{m}^{4}+J_{m, s}\right) \ddot{\phi}_{m}+T_{m, \text { out }}\right)^{2} d t}
\end{gathered}
$$

Solving (27), for the DC-motor length, $l_{m}$, results in a function of $l_{m}$ which depends on stator radius, $r_{m}$. Using (27) with another form factor inequality constraint given in (28), the DC-motor is dimensioned and its volume can be determined.

$$
0.5 \leq \frac{l_{m}}{r_{m}} \leq 5
$$

Another constraint is on the maximum allowed speed of the DC-motor, $w_{m, \text { peak }}$, which has to be higher than or equal to the speed requirements of the load.

$$
w_{m, \text { peak }} \geq \max \left|\dot{\phi}_{l}\right| n
$$

The fulfillment of (28) and (29) assures that the DC-motor is able to drive under the defined load specifications. Equation (29) can be reformulated to express the maximum allowed gear ratios.

The main constraint on the gear is bending stress in the root of a gear teeth and Hertzian pressure on the teeth contact surfaces. If the number of the sun gear teeth is small and/or the gear is made from ductile steel, the Hertzian pressure is the constraining factor for the design of the gears. Every tooth surface needs to fulfill [20]

$$
r_{\mathrm{g}}^{2} b_{g} \geq Z_{\mathrm{H}}^{2} Z_{\mathrm{M}}^{2} Z_{\epsilon} K_{\mathrm{H} \alpha} K_{\mathrm{H} \beta} \frac{T_{g, \text { out }}(n-1)^{2}}{6(n-2) \sigma_{\mathrm{H}, \max }^{2}}
$$

where $Z_{H}, Z_{M}, Z_{\epsilon}, K_{H \alpha}$ and $K_{H \beta}$ are parameters with minor impact on the system and therefore can be considered independent of $n$. We have two more inequality and equality constraints to ensure the physical design of the gear as in (31) and (32),

$$
\begin{gathered}
0.05 \leq \frac{b_{g}}{r_{g}} \leq 4 \\
b_{c}=0.5 b_{g}
\end{gathered}
$$

where $b_{g}$ is the total width of the gear, $b_{c}$ is the carrier width and $r_{g}$ is the planetary gear radius.

The dimensioning model for the shaft is from classic solid mechanics. The following equations describe shaft properties,

$$
\begin{gathered}
r_{s h}=\sqrt[3]{\frac{2 \hat{T}_{\text {sh,out }}}{\tau_{\text {sh,max }} \pi}} \\
J_{s h}=\frac{\pi r_{s h}^{4}}{2} \\
G=\frac{E}{2(1+\nu)} \\
k_{s h}=\frac{G J_{s h}}{l_{s h}}
\end{gathered}
$$


where $r_{s h}$ is shaft radius, $\hat{T}_{\text {sh,out }}$ is the peak transferred torque, $\tau_{s h, \max }$ is the maximum permissible shear stress, $G$ is shear modulus, $E$ is Young's module, $\nu$ is Poisson's ratio, $k_{s h}$ is the shaft stiffness [21], [22].

\section{3) Control constraints of the optimization:}

While designing the control, stability of the system as an optimization constraint should be verified. Satisfaction of this property means a non-infinite value for the step response.

To avoid the aliasing effect in sampling of signals, we must set an optimization constraint on the sampling time, $T_{s}$. A good sampling time should be in a defined range such as in (37).

$$
5 w_{c} \leqslant \frac{2 \pi}{T_{s}} \leqslant 100 w_{c}
$$

where $w_{c}$ is the desired bandwidth of the continuous time closed loop system.

Defining the sampling frequency, $w_{s}$, as the second optimization function aims at reducing computational costs. On the other hand, a too low sampling frequency deteriorates control performance. To avoid this problem, control performance constraint(s) are considered. Integrated square error $(I S E)$ and maximum error $(\max (e r))$ are defined as control constraints on the optimization problem.

$$
\begin{gathered}
I S E=\sqrt{\sum_{0}^{m}\left(r_{\text {des }}[m]-y_{\text {out }}[m]\right)^{2}} \\
\max (e r)=\max \left(\left|r_{\text {des }}[m]-y_{\text {out }}[m]\right|\right)
\end{gathered}
$$

where $r_{\text {des }}$ and $y_{\text {out }}$ are the desired reference input and the controlled output.

\section{CASE STUDY}

The case study is a mechatronic system including a DCmotor, one gear stage, a flexible shaft and a load component as shown in Fig. 3. Friction, external disturbances and measurement noise are not considered. A load component is a mechanical body with a defined inertia, $J_{l}$.

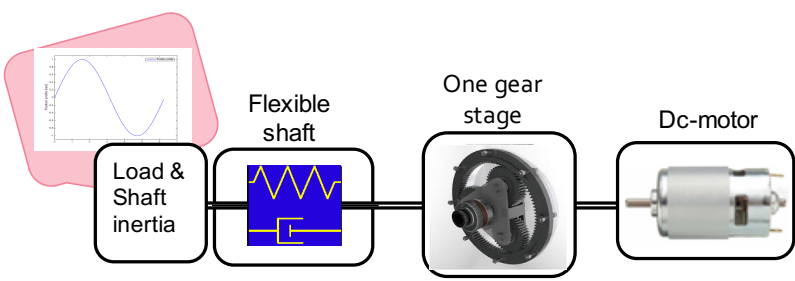

Fig. 3. A Mechatronics case study

The system is optimized and simulated for trapezoidal and sinusoidal load profiles shown in Fig. 4 and Fig. 5, respectively. $T_{\text {peak }}$ and $T_{r m s}$ are the peak and root mean square of the torques which the physical system is designed for.
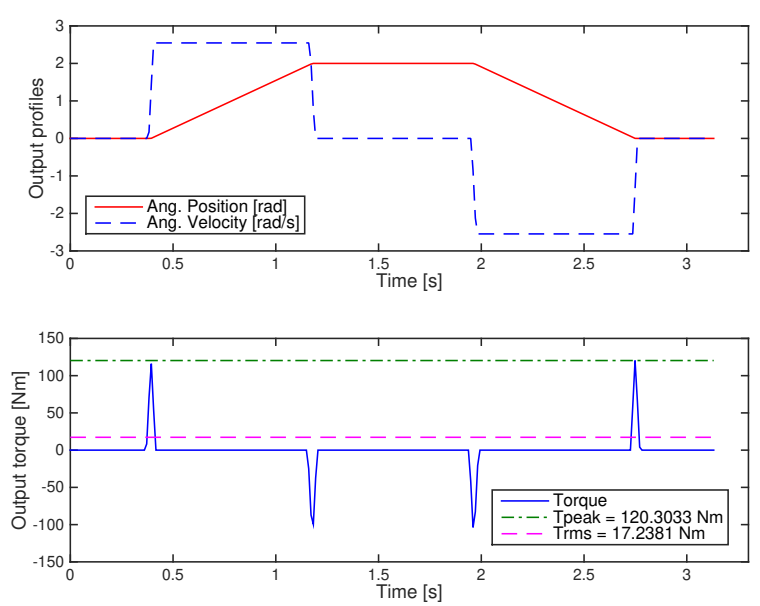

Fig. 4. Trapezoidal (load) profile
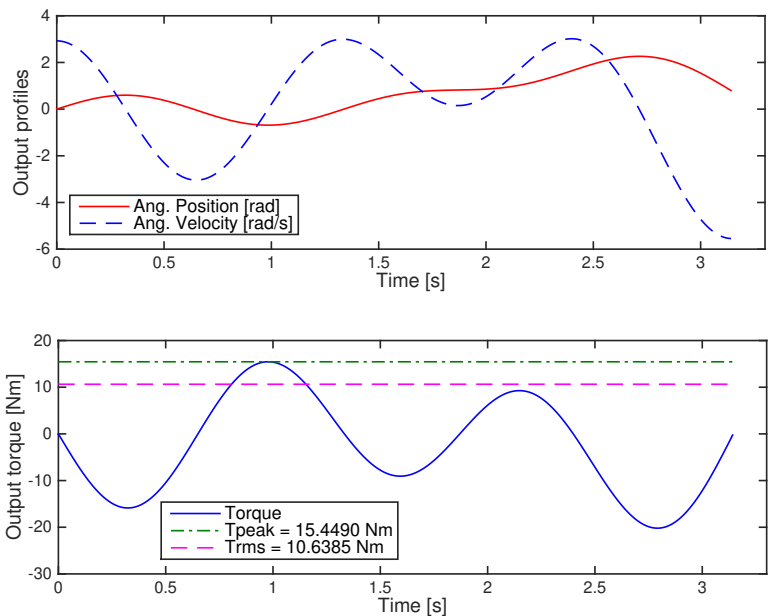

Fig. 5. Sinusoidal (load) profile

In previous studies published by the authors [5], [6], [7], [8], and [21], the impact of the actual dynamic load on the physical design of each component is neglected. In this study, the influence of the actual controlled output i.e. actual output torque profile on the dimensioning of each component is considered as an extra optimization constraint. In order to evaluate this, the satisfaction of the physical constraints of each component in the optimization loop is assessed for each individual using the actual RMS torque and actual peak torque that are derived from actual state variables in between components. The actual computed output torque and velocity with the actual $T_{\text {peak }}$ and $T_{r m s}$ values for the trapezoidal and sinusoidal load profiles are depicted in Fig. 6 and Fig. 7, respectively.

As discussed earlier the main purpose of this paper is to extend a previously published method by the authors to cover gaps in the integration of physical design and control 

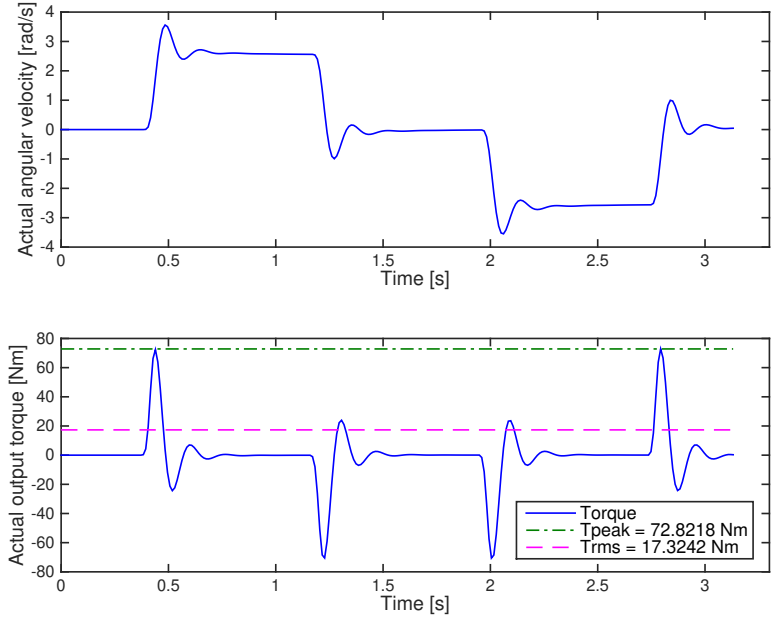

Fig. 6. Actual output velocity and torque for trapezoidal profile
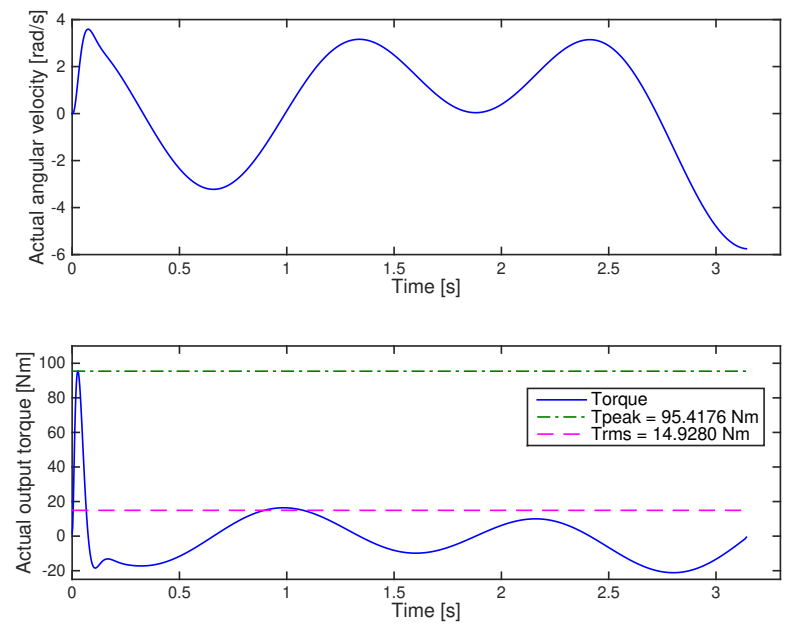

Fig. 7. Actual output velocity and torque for sinusoidal profile

implementation optimization. Therefore, the effect of control constraints on the physical design and control implementation with focus on the sampling time is investigated. The system is optimized and simulated for two different output profiles for a defined fixed gear ratio of 50 to study dependencies between physical, control and implementation parameters. The optimization problem is formulated as follows.

$\min$

$$
\begin{aligned}
& o b j=k_{1}\left(\pi r_{\mathrm{m}}^{2} l_{\mathrm{m}}+\pi r_{\mathrm{g}}^{2}\left(b_{g}+b_{c}\right)+\pi r_{s h}^{2} l_{s h}\right) \\
& +k_{2}\left(2 \pi / T_{s}\right)
\end{aligned}
$$

s.t.

$$
\begin{aligned}
& 0.04 \leq l_{m} \leq 0.12 \\
& 0.5 \leq \frac{l_{m}}{r_{m}} \leq 5 \\
& w_{m, p e a k} \geq \max \left|\dot{\phi}_{l}\right| n \\
& 0.02 \leq r_{g} \leq 0.1 \\
& 0.05 \leq l_{s h} \leq 0.12 \\
& 0.05 \leq \frac{b_{g}}{r_{g}} \leq 4 \\
& k_{s h}=\frac{G J_{s h}}{l_{s h}} \\
& 2 \leq c_{b} \leq 30 \\
& 5 w_{c} \leq \frac{2 \pi}{T_{s}} \leq 100 w_{c} \\
& 1 \leq k_{p} \leq 300 \\
& 1 \leq k_{d} \leq 300 \\
& 1 \leq r_{0} \leq 300 \\
& \max \left(\left|r_{\text {des }}[n]-y_{\text {out }}[n]\right|\right) \leq b
\end{aligned}
$$

where $b$ is a boundary for the control performance constraint, $\max (e r)$. As seen from the above optimization problem, the sampling rate of the system $\left(w_{s}\right)$ as a second objective function is a function of $T_{s}$ and hence depends on $w_{b}, c o$ and $w_{c}$ according to (17) and (37). The values for $w_{b}$ and $w_{c}$ are influenced by the control design parameters and the physical design parameters which lead to an implicit impact on the sampling frequency $\left(w_{s}\right)$.

For the GA itself, some parameters are varied until they do not affect the results of the objectives. These parameters are the tolerance function ( $\mathrm{Tol})$, number of generations $(\mathrm{Gen})$ and the stall generation limit $(S g l)$. The variations used are as follows, $T o l=1 e^{-3}, 1 e^{-4}, 1 e^{-5}, 1 e^{-6}, \mathrm{Gen}=150,200,300$, and $S g l=100,150,200$ each at a time. The finally chosen values in all tests for these parameters are $T o l=1 e^{-4}, \mathrm{Gen}=$ 300 , and $S g l=100$, respectively.

The parameter boundaries for physical constraints are defined based on basic mechanical engineering principles. The ranges for PD control parameters are selected by trial and error. The free design variables and fixed parameters for all of the tests are given in Table I and II. The material properties of components are derived by considering steel material. The estimated damping, $d_{s h}$, captures material damping in shaft and viscous friction in bearings.

\section{Discussion}

\section{A. Control Performance Constraint Impact on the Design}

The system is designed and optimized for smallest volume and slowest sampling frequency by varying the boundaries of the control constraint $\max (\mathrm{er})$. The results of the design for the trapezoidal and sinusoidal profiles as shown in Fig. 4 and Fig. 5 are presented in Table III and Table IV, respectively. Analysing the results clarifies that the relation between the control constraint $\max (e r)$ and $w_{s}$ and $w_{c}$ follow a pattern 
TABLE I

DESIGN VARIABLES

\begin{tabular}{|c|c|c|}
\hline Variables & Ranges & Physical definition \\
\hline$l m$ & {$[0.04-0.12](m)$} & Length of the DC-motor \\
\hline$r g$ & {$[0.02-0.1](m)$} & Radius of the gear \\
\hline$l_{s h}$ & {$[0.05-0.12](m)$} & Length of shaft \\
\hline$c_{b}$ & {$[2-30]$} & Sampling rate coefficient \\
\hline$k_{p}$ & {$[1-300]$} & Proportional term of $P D$ \\
\hline$k_{d}$ & {$[1-300]$} & Derivative term of $P D$ \\
\hline$r_{0}$ & {$[1-300]$} & low pass filter \\
\hline
\end{tabular}

TABLE II

DESIGN PARAMETERS

\begin{tabular}{|c|c|c|}
\hline Variables & Fixed value & Physical definition \\
\hline$r m$ & $0.03(\mathrm{~m})$ & Radius of the DC-motor \\
\hline$b g$ & $0.05(\mathrm{~m})$ & Width of the gear \\
\hline$r_{s h}$ & $0.003(\mathrm{~m})$ & Radius of shaft \\
\hline$d_{s h}$ & $2(\mathrm{Nms} / \mathrm{rad})$ & Damping \\
\hline
\end{tabular}

(shown in Fig. 8) as expected (control theory). The changes for volume value are not considerable. We can see from Fig. 8 that by increasing the control constraint boundaries, the bandwidth of the closed loop system decreases, thus the sampling frequency decreases.
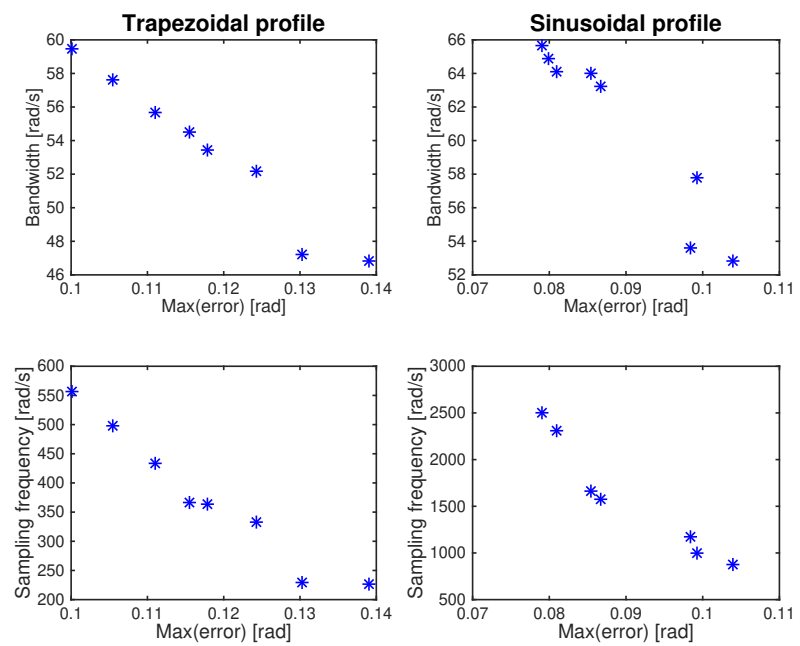

Fig. 8. The pattern for bandwidth and sampling frequency over $\max (\mathrm{er})$ for trapezoidal profile

The physical constraints of the system are checked over static output torque and dynamic output torque which limit the solution space for the physical design. Comparing numerical results in Table III and Table IV, it is clear for both load profiles that a lower (more optimal) sampling frequency is possible with a more loose definition of control constraint boundaries. More investigations are needed to clarify how the control constraint boundaries affect system volume.

The results for $c_{b}$ in Table III and Table IV show that the sampling frequency, $w_{s}$, of the system satisfies the basic requirement for embedded control implementation as being at least two times the highest frequency of the system.

\section{B. Control Performance Evaluation}

The control performance of the optimal $P D$ control is verified by the position tracking of the output shaft. For the evaluated Test 1 and Test 9 in Table III and Table IV, the trajectory tracking performance of the output shaft angle for trapezoidal and sinusoidal profiles are presented in Fig. 9, Fig. 10 and Fig. 11, respectively. The control performance of the optimal $P D$ method satisfies the control constraint by adequately tracking the reference input.
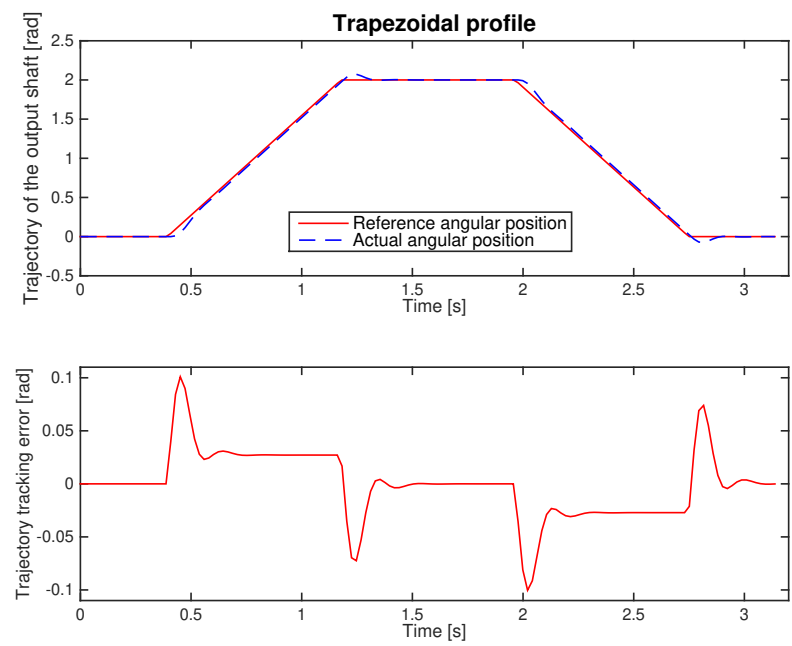

Fig. 9. Trajectory tracking performance of the output shaft (trapezoidal load profile)

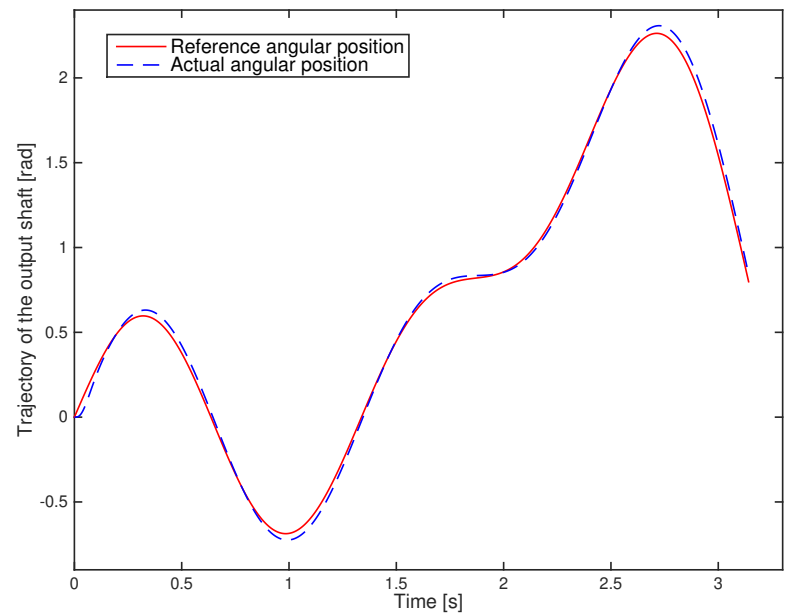

Fig. 10. Trajectory tracking performance for the sinusoidal load profile

\section{Verification in Simulink}

Test 9 in Table IV is modeled in Simulink with the derived optimization parameters for the physical design and control 
TABLE III

RESULTS OF THE GA OPTIMIZATION FOR DIFFERENT ERROR RANGES FOR THE TRAPEZOIDAL LOAD PROFILE $(n=50)$

\begin{tabular}{|c|c|c|c|c|c|c|c|c|c|c|c|c|}
\hline Test & $l_{m}[m]$ & $c_{b}$ & $r_{g}$ & $l_{s h}$ & $\max (e r)$ & $v\left[m^{3}\right]$ & $w_{s}[\mathrm{rad} / \mathrm{s}]$ & $T_{s}[s]$ & $w_{c}$ & $P$ & $D$ & $r 0$ \\
\hline 1 & 0.1112 & 4.6 & 0.0897 & 0.1071 & $0.1 \leq 0.1$ & 0.0022 & 557.9 & 0.0113 & 59.48 & 17.4 & 162.1 & 33.3 \\
\hline 2 & 0.1118 & 4.4 & 0.0905 & 0.1068 & $0.105 \leq 0.105$ & 0.0022 & 497.4 & 0.0126 & 57.58 & 16.2 & 150.6 & 31.6 \\
\hline 3 & 0.1125 & 4.2 & 0.0912 & 0.1069 & $0.11 \leq 0.11$ & 0.0023 & 433.1 & 0.0145 & 55.69 & 14.98 & 138.4 & 28.8 \\
\hline 4 & 0.1128 & 4.1 & 0.0916 & 0.1033 & $0.115 \leq 0.115$ & 0.0023 & 366.41 & 0.0171 & 54.53 & 14.4 & 132.4 & 28.1 \\
\hline 5 & 0.1129 & 4.07 & 0.0917 & 0.1041 & $0.1178 \leq 0.12$ & 0.0023 & 362.6 & 0.0173 & 53.47 & 14.2 & 129.9 & 29.5 \\
\hline 6 & 0.1136 & 3.9 & 0.0926 & 0.1074 & $0.1242 \leq 0.125$ & 0.0023 & 333.3 & 0.0189 & 52.14 & 12.86 & 117.7 & 23.85 \\
\hline 7 & 0.066 & 2.1 & 0.0874 & 0.0841 & $0.13 \leq 0.13$ & 0.002 & 228.7 & 0.0275 & 47.2 & 15.8 & 113.8 & 32.9 \\
\hline 8 & 0.0606 & 3.02 & 0.0968 & 0.1194 & $0.139 \leq 0.14$ & 0.0024 & 226 & 0.0278 & 46.87 & 22.5 & 76.9 & 16.2 \\
\hline
\end{tabular}

TABLE IV

RESULTS OF THE GA OPTIMIZATION FOR DIFFERENT ERROR RANGES USING SINUSOIDAL LOAD PROFILE $(n=50)$

\begin{tabular}{|c|c|c|c|c|c|c|c|c|c|c|c|c|}
\hline Test & $l_{m}[m]$ & $c_{b}$ & $r_{g}$ & $l_{s h}$ & $\max (e r)$ & $v\left[m^{3}\right]$ & $w_{s}[\mathrm{rad} / \mathrm{s}]$ & $T_{s}[s]$ & $w_{c}$ & $P$ & $D$ & $r 0$ \\
\hline 9 & 0.0938 & 7.6 & 0.0737 & 0.089 & $0.079 \leq 0.079$ & 0.0015 & 2500 & 0.0025 & 65.65 & 35.5 & 296.5 & 70.6 \\
\hline 10 & 0.0942 & 7.5 & 0.074 & 0.0888 & $0.0798 \leq 0.0798$ & 0.0016 & 2420 & 0.0026 & 64.92 & 35 & 292.3 & 70.7 \\
\hline 11 & 0.0845 & 7.4 & 0.0745 & 0.0883 & $0.08 \leq 0.08$ & 0.0016 & 2310 & 0.0027 & 64.1 & 34.4 & 286.4 & 70.4 \\
\hline 12 & 0.0977 & 6.74 & 0.0776 & 0.0812 & $0.085 \leq 0.085$ & 0.0017 & 1655 & 0.0038 & 63.99 & 30 & 250.4 & 59.4 \\
\hline 13 & 0.0982 & 6.6 & 0.0781 & 0.0805 & $0.086 \overline{7} \leq 0.09$ & 0.0017 & 1571 & 0.004 & 63.3 & 29.3 & 244.3 & 58.6 \\
\hline 14 & 0.1011 & 6.0 & 0.081 & 0.0812 & $0.098 \leq 0.098$ & 0.0018 & 1166 & 0.0054 & 53.64 & 25.1 & 208.4 & 63.0 \\
\hline 15 & 0.103 & 5.9 & 0.0829 & 0.0855 & $0.0993 \leq 0.1$ & 0.0019 & 1000 & 0.0063 & 57.79 & 23.4 & 192.7 & 50.5 \\
\hline 16 & 0.1046 & 6.0 & 0.0846 & 0.0876 & $0.11 \leq 0.11$ & 0.002 & 930.2 & 0.0068 & 46.58 & 22.4 & 171.9 & 62.8 \\
\hline
\end{tabular}

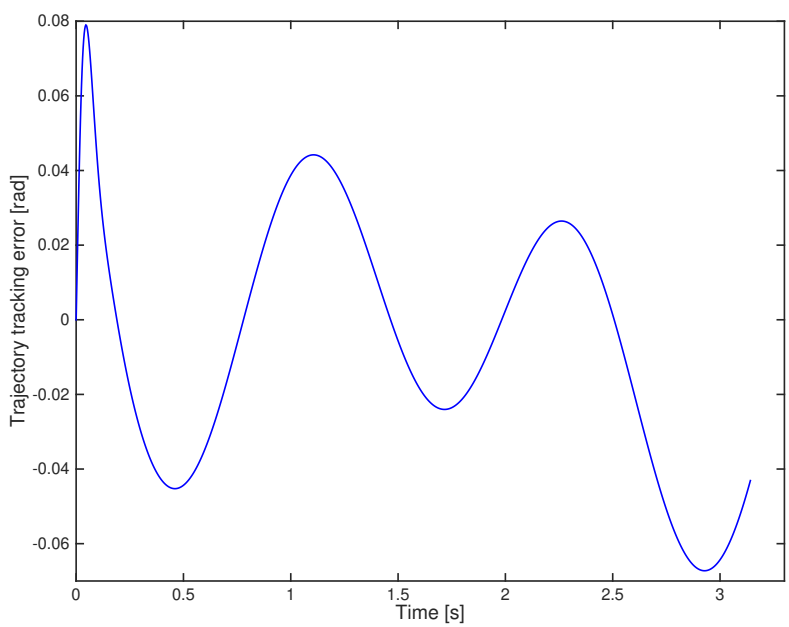

Fig. 11. Trajectory tracking error for the sinusoidal load profile

system to validate the correctness of the IDIOM framework models, computations and simulations. The trajectory tracking performance of the output shaft and the error are evaluated and shown in Fig. 12 and Fig. 13 which correspond to the results depicted in Fig. 10 and Fig. 11 which are derived in the IDIOM framework using the methodology presented in this paper. The CAD model for Test 9 in Table IV is shown in Fig. 14.

\section{Comparisons with Previous Method}

The presented method is compared to the published method presented in previous studies [5], [8], [21] that focused on optimizing the system for one primary objective, for example the size of the system in terms of volume. In the extended method which is labeled as 'new' in Table V and Table VI,

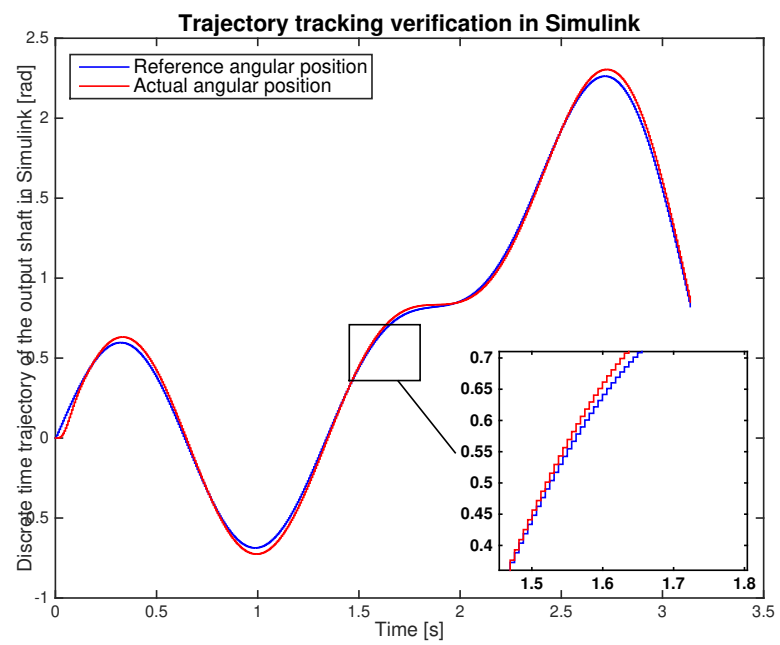

Fig. 12. Verification of the discrete time trajectory tracking for the output shaft in Simulink

both volume and sampling frequency of the system are optimized. For as fair comparison as possible the input trajectories are made equal, i.e. the reference trajectory in the continuous time method (old) is sampled with the same frequency as is used in the 'new' method. The optimization objective in the 'old' method is defined to be only volume.

It is expected that a discrete time system imposes restrictions on the design compared to a continuous time system due to the additional constraints and objective. Even though the sampling period is sufficiently small, the 'old' method results in a smaller system size. This is intuitively reasonable since only one objective function has to be considered. 
TABLE V

COMPARISIONS OF NEW METHOD AND OLD METHOD FOR THE TRAPEZOIDAL PROFILE $(n=50)$

\begin{tabular}{|c|c|c|c|c|c|c|c|c|c|c|c|c|}
\hline Test & $l_{m}[m]$ & $c_{b}$ & $r_{g}$ & $l_{s h}$ & $\max (e r)$ & $v\left[m^{3}\right]$ & $w_{s}[\mathrm{rad} / \mathrm{s}]$ & $T_{s}[s]$ & $w_{c}$ & $P$ & $D$ & $r 0$ \\
\hline new & 0.1112 & 4.6 & 0.0897 & 0.1071 & $0.1 \leq 0.1$ & 0.0022 & 557.9 & 0.0113 & 59.48 & 17.4 & 162.1 & 33.3 \\
\hline old & 0.0488 & - & 0.072 & 0.0484 & $0.0429 \leq 0.1$ & 0.0014 & - & 0.0113 & 101.8364 & 223.8 & 261.7 & 36.1 \\
\hline
\end{tabular}

TABLE VI

COMPARISIONS OF NEW METHOD AND OLD METHOD FOR THE SINUSOIDAL PROFILE $(n=50)$

\begin{tabular}{|c|c|c|c|c|c|c|c|c|c|c|c|c|}
\hline Test & $l_{m}[\mathrm{~m}]$ & $c_{b}$ & $r_{g}$ & $l_{s h}$ & $\max (\mathrm{er})$ & $v\left[\mathrm{~m}^{3}\right]$ & $w_{s}[\mathrm{rad} / \mathrm{s}]$ & $T_{s}[s]$ & $w_{c}$ & $P$ & $D$ & $r 0$ \\
\hline new & 0.0845 & 7.4 & 0.0745 & 0.0883 & $0.08 \leq 0.08$ & 0.0016 & 2310 & 0.0027 & 64.1 & 34.4 & 286.4 & 70.4 \\
\hline old & 0.0448 & - & 0.0758 & 0.0421 & $0.0626 \leq 0.8$ & 0.0015 & - & 0.0027 & 78.9 & 196.6 & 140.0 & 69.1 \\
\hline
\end{tabular}

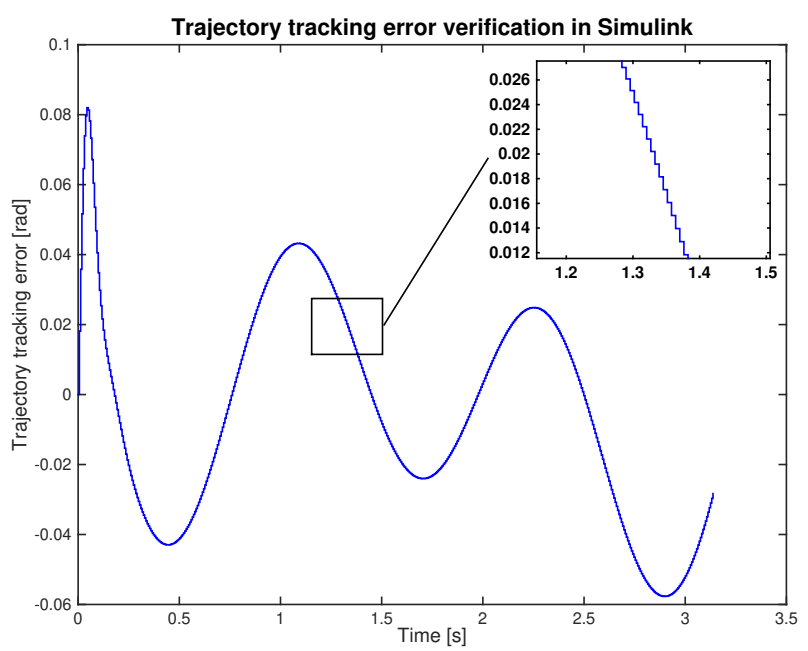

Fig. 13. Verification of the discrete time trajectory tracking error in Simulink

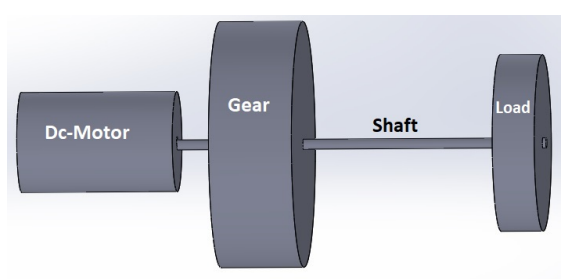

Fig. 14. CAD model using optimal parameters

\section{E. Weight Factors Impact on the Co-Design}

The weight factors $k_{1}$ and $k_{2}$ for the weighted sum function using the pareto optimal front set are varied considering $k_{1}+$ $k_{2}=1$ and the resulting $v$ and $w_{s}$ are compared. As presented in Table VII, we can see that the lower the weight factor is defined the lower the importance of that objective is i.e., larger value of any of the weight factors improves the optimization result for that objective.

\section{F. Gear Ratio Impact on the Co-Design}

The planetary gear is a core component in physical and control optimization of mechatronic systems. Thus, the effect of gear ratio on the optimization and control performance
TABLE VII

RESULTS OF THE GA OPTIMIZATION FOR DIFFERENT WEIGTH FACTORS OF PARETO FRONT FUNCTION USING SINUSOIDAL PROFILE $(n=70)$

\begin{tabular}{|c|c|c|c|}
\hline$k_{1}$ & $k_{2}$ & $v\left[\mathrm{~cm}^{3}\right]$ & $w_{s}[\mathrm{rad} / \mathrm{s}]$ \\
\hline 0.1 & 0.9 & 1907.8 & 288.6 \\
\hline 0.2 & 0.8 & 1852.6 & 295.3 \\
\hline 0.3 & 0.7 & 1585.8 & 399.5 \\
\hline 0.35 & 0.65 & 1445 & 467.4 \\
\hline 0.4 & 0.6 & 1378.3 & 511.2 \\
\hline 0.6 & 0.4 & 1314.3 & 558.9 \\
\hline 0.8 & 0.2 & 1290.1 & 632.4 \\
\hline 0.9 & 0.1 & 1234.3 & 925.6 \\
\hline 0.95 & 0.05 & 1222.8 & 1082.5 \\
\hline 0.99 & 0.01 & 1222.1 & 1123 \\
\hline 0.995 & 0.005 & 1221 & 1289.1 \\
\hline 0.9999 & 0.001 & 1220 & 1486 \\
\hline
\end{tabular}

is investigated. The system is optimized for a few defined fixed values of gear ratio using the trapezoidal load profile. Fig. 15 depicts the result before implementing the weighted sum function on the pareto optimal front set for these tests. The results depict that the control performance improves with gear ratio that is in line with results presented by Roos in [20] i.e. it is possible to decrease control constraint boundaries by increasing the gear ratio. This is due to an increase of the torque margin with gear ratio. The decrease in $\max (e r)$ requires an increase in bandwidth of the closed loop system. Hence, the sampling frequency of the system is increased. These results show that larger values of $n$ results in an optimized system without an unnecessarily small sampling time causing computational cost. Moreover, larger values of $n$ result in a better control performance from control constraints perspective. In further studies it should be considered to have $n$ as a free design variable during the optimization. Fig. 15 also indicates strong influence of $k_{1}$ and $k_{2}$ on the result of the final optimized system for each value of $n$.

\section{EXTRA ANALYSIS}

The main focus here is on further analysing the optimum results, paretofront optimum set and the sum weigthing function. In the previous analysis, the impact of first objective that was volume on the final optimum result is neglected due to the uneven range of the two objectives. Considering the definition of weight factors, if $k_{1}$ and $k_{2}$ are positive, minimizing $o b j$ 

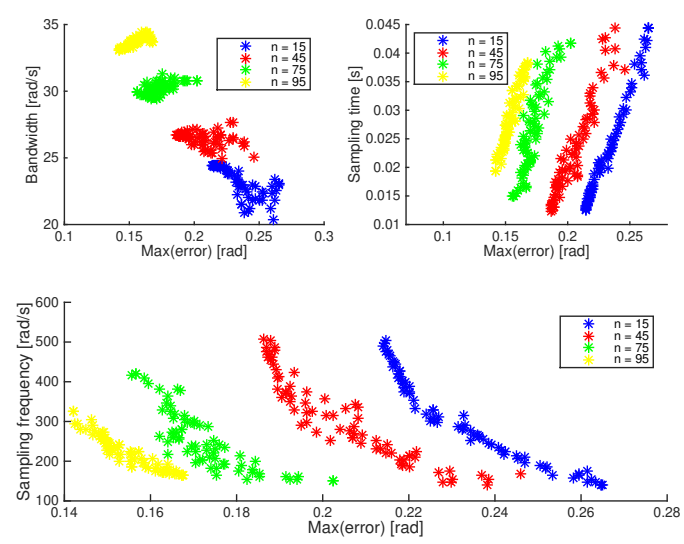

Fig. 15. Different gear ratio analysis

yields in paret optimality solution. However, it is still difficult to decide the weights to indicate the relative importance of each objective due to the difference in objectives magnitudes. One proper method to select the weight factors is to normalize the objectives. This is done using (40) and (41) for minimizing and maximizing objectives, respectively [4].

$$
\begin{gathered}
o b j_{i}^{n o r m}=\frac{\max \left(o b j_{i}\right)-o b j_{i}}{\max \left(o b j_{i}\right)-\min \left(o b j_{i}\right)} \\
o b j_{i}^{\text {norm }}=1-\frac{\max \left(o b j_{i}\right)-o b j_{i}}{\max \left(o b j_{i}\right)-\min \left(o b j_{i}\right)}
\end{gathered}
$$

Normallizing objectives result in a range between zero and one for all of the objectives, hence, the selection of weigth factors based on importance of the relevant objective becomes more realistic. To do this further analysis, a few extra tests are performed that are presented in Table VIII, the gear ratio, $n$, for these tests are selected to be fixed as 40 and the shaft damping, $d_{s h}$ is chosen to be 8 ,

Discussed previous results hold for the results given in Table VIII where the weighting sum function is applied on the normallized objectives, so, both of the objectives have exact the same importance in these tests. For comparison purposes and in-depth study of the weigth factors impact on the design parameters and objectives, we have defined $k_{1}=0.9$ and $k_{2}=0.1$ and $k_{1}=0.1$ and $k_{2}=0.9$ in Table IX and Table X, respectively. These choices are just made based on the importance of the objectives after normalizing them so the scale of the two objectives are the same. The tests that are showing some major changes are presented in these Tables.

Checking the impact of weigth factors $k_{1}$ and $k_{2}$ on the physical parameters individually we see in Table IX, by defining $k_{1}=0.1$ and $k_{2}=0.9$, the final optimized result after applying these factors for $w_{s}$ is better (minimized) than volume (minimizing $w_{s}$ is prior to minimizing $v$ ). This means that by comparing each test in Table VIII and Table IX, volume should be larger in Table IX but $w_{s}$ should be smaller (we see this in all tests). What is dominant in volume calculation out of physical components as can be seen from comparing Tables
VIII-IX is the length of motor that is larger in the presented tests in Table IX than Table VIII due to the less importance of volume objective and is smaller in Table X. Sampling frequency, $w_{s}$, on the other hand, should be smaller in Table IX than in Table VIII for each test. This affects physical design parameters, i.e., smaller $w_{s}$ means smaller natural frequency and smaller eigenvalues in the system (indirect impact on the physical design parameters), which leads to a larger length of the shaft, $l_{s h}$, and therefore, volume of the shaft (we see this by comparing each test in Table VIII and Table IX).

$$
\uparrow k 2 \rightarrow \downarrow w_{s} \rightarrow \downarrow \text { natural frequency } \rightarrow \downarrow w_{b} \rightarrow \uparrow l_{\text {sh }}
$$

All the above is opposite when comparing Tables VIII-X.

$$
\downarrow k 2 \rightarrow \uparrow w_{s} \rightarrow \uparrow \text { natural frequency } \rightarrow \uparrow w_{b} \rightarrow \downarrow l_{\text {sh }}
$$

By comparing each test in three Tables, we see that as far as the boundary for the control constraint $\max (e r)$ is increases it allows larger solution space for both of the objectives.

\section{CONCLUSION}

Disregarding the impact of control implementation in optimization of mechatronic systems might result in overdimensioning in one or more of the involved engineering domains. Hence, an integrated design optimization approach is extended to start covering some of the current gaps in closed loop mechatronic system design. The method is improved to integrate physical design optimization and control implementation optimization. A multi-objective optimization method is utilized and an optimal PD control method with a low-pass filter is implemented. The approach is applied to a mechatronic case study with two different load profiles and a few test cases are examined to investigate relations between physical, control and implementation parameters. To deepen the understanding of relations between domains further studies are needed. Additional free design variables such as for example the gear ratio, motor radius and shaft radius should be investigated. Additional embedded system implementation aspects should also be investigated, such as time delays and sensor resolutions. The now extended IDIOM framework facilitates such further studies. The IDIOM approach includes physical design optimization, control design optimization and the coupling between control implementation and design and has a broader coverage in comparison with the other presented methods and tools in [13], [23], [24], [25], [26]. The method is compared with a previously published method that was focused on optimizing one primary objective and the advantage of the current method is highlighted. The optimization approach is tested for different weight factors of a scalar weighted sum objective function based on the pareto front and the impact of each parameter on the objectives are studied. The influence of different gear ratios as a main parameter in the design optimization is investigated. 
TABLE VIII

RESULTS OF THE GA OPTIMIZATION FOR DIFFERENT ERROR RANGES FOR THE TRAPEZOIDAL LOAD PROFILE $\left(k_{1}=0.5, k_{2}=0.5\right)$

\begin{tabular}{|c|c|c|c|c|c|c|c|c|c|c|c|c|}
\hline Test & $l_{m}[\mathrm{~m}]$ & $r_{g}$ & $c_{b}$ & $l_{s h}$ & $\max (\mathrm{er})$ & $v\left[\mathrm{~cm}^{3}\right]$ & $w_{s}[\mathrm{rad} / \mathrm{s}]$ & $T_{s}[s]$ & $w_{b}$ & $k_{p}$ & $k_{d}$ & $r_{0}$ \\
\hline 1 & 0.081 & 0.0544 & 3.0 & 0.0714 & $0.11 \leq 0.11$ & 930.85 & 298.79 & 0.021 & 99.13 & 58.5 & 63.2 & 37.2 \\
\hline 2 & 0.0864 & 0.0503 & 2.0 & 0.0452 & $0.1196 \leq 0.12$ & 844.03 & 244.21 & 0.0257 & 121.2 & 54.1 & 43.6 & 30.4 \\
\hline 3 & 0.0931 & 0.0424 & 2.2 & 0.0593 & $0.13 \leq 0.13$ & 691.5 & 228.02 & 0.0276 & 102.6 & 32.2 & 59.8 & 43.2 \\
\hline 4 & 0.091 & 0.0391 & 2.5 & 0.0894 & $0.138 \leq 0.14$ & 625.1 & 215.93 & 0.0291 & 84.44 & 30.5 & 60.3 & 56.95 \\
\hline
\end{tabular}

TABLE IX

RESULTS OF THE GA OPTIMIZATION FOR DIFFERENT ERROR RANGES FOR THE TRAPEZOIDAL LOAD PROFILE $\left(k_{1}=0.1, k_{2}=0.9\right)$

\begin{tabular}{|c|c|c|c|c|c|c|c|c|c|c|c|c|}
\hline Test & $l_{m}[\mathrm{~m}]$ & $r_{g}$ & $c_{b}$ & $l_{s h}$ & $\max (\mathrm{er})$ & $v\left[\mathrm{~cm}^{3}\right]$ & $w_{s}[\mathrm{rad} / \mathrm{s}]$ & $T_{s}[\mathrm{~s}]$ & $w_{b}$ & $k_{p}$ & $k_{d}$ & $r_{0}$ \\
\hline 1 & 0.0826 & 0.0544 & 3.0 & 0.0715 & $0.1094 \leq 0.11$ & 935.54 & 295.89 & 0.0212 & 98.17 & 58.2 & 63.3 & 37.5 \\
\hline 2 & 0.0865 & 0.0503 & 2.0 & 0.0453 & $0.1196 \leq 0.12$ & 845.03 & 243.21 & 0.0258 & 121.8 & 53.9 & 43.6 & 31.4 \\
\hline 3 & 0.0932 & 0.0424 & 2.2 & 0.0594 & $0.13 \leq 0.13$ & 691.9 & 227.9 & 0.0277 & 102.5 & 32.5 & 59.9 & 43.8 \\
\hline 4 & 0.0912 & 0.0391 & 2.6 & 0.0896 & $0.138 \leq 0.14$ & 626 & 215.7 & 0.0291 & 84.25 & 30.2 & 62.2 & 57.7 \\
\hline
\end{tabular}

TABLE X

RESULTS OF THE GA OPTIMIZATION FOR DIFFERENT ERROR RANGES FOR THE TRAPEZOIDAL LOAD PROFILE $\left(k_{1}=0.9, k_{2}=0.1\right)$

\begin{tabular}{|c|c|c|c|c|c|c|c|c|c|c|c|c|}
\hline Test & $l_{m}[\mathrm{~m}]$ & $r_{g}$ & $c_{b}$ & $l_{s h}$ & $\max (\mathrm{er})$ & $v\left[\mathrm{~cm}^{3}\right]$ & $w_{s}[\mathrm{rad} / \mathrm{s}]$ & $T_{s}[s]$ & $w_{b}$ & $k_{p}$ & $k_{d}$ & $r_{0}$ \\
\hline 1 & 0.0752 & 0.0544 & 3.0 & 0.0655 & $0.11 \leq 0.11$ & 914.13 & 324.17 & 0.0194 & 106.9 & 59.0 & 61.8 & 38.0 \\
\hline 2 & 0.0863 & 0.0503 & 2.0 & 0.0446 & $0.1188 \leq 0.12$ & 843.8 & 245.3 & 0.0256 & 122.0 & 54.8 & 43.6 & 30.5 \\
\hline 3 & 0.0931 & 0.0424 & 2.2 & 0.0593 & $0.13 \leq 0.13$ & 691.5 & 228.1 & 0.0275 & 102.6 & 32.1 & 60.1 & 43.5 \\
\hline 4 & 0.0792 & 0.0392 & 2.5 & 0.058 & $0.138 \leq 0.14$ & 589.9 & 279.95 & 0.0224 & 111.52 & 39.7 & 60.7 & 56.3 \\
\hline
\end{tabular}

\section{REFERENCES}

[1] R. Isermann, "Mechatronic systems-Innovative products with embedded control," Control Engineering Practice, vol. 16(1), pp.14-29, 2008.

[2] B. Wittenmark, J. Nilsson, and M. Törngren, "Timing Problems in Real-Time Control Systems," In: Proceedings of the American Control Conference. pp. 2000-2004, 1995.

[3] K. E. Årzen, A. Cervin, J. Eker, and L. Sha., "An Introduction to Control and Scheduling Co-Design," In: Proceedings of the 39th IEEE Conference on Decision and Control., Sydney, Australia, pp. 4865-4870, 2000.

[4] A. Aminifar, P. Eles, Z. Peng, A. Cervin, K. E. Årzén, "Control-Quality Driven Design of Embedded Control Systems with Stability Guarantees," IEEE Design and Test vol. 35, pp.38-46, 2017.

[5] F. Rahimi, L. Feng, J. Wikander, and D. Frede, "Early Phase DesignOptimization of Mechatronic Systems," Proceedings of the 5th International Conference on Control, Mechatronics and Automation, Edmonton, AB, Canada, pp.42-49, 2017.

[6] D.Malmquist, D. Frede, J. Wikander, "Holistic design methodology for mechatronic systems," Proceedings of the Institution of Mechanical Engineers, Part I: Journal of Systems and Control Engineering, vol. 228(10), pp. 741-757, 2014.

[7] D. Frede, D. Malmquist, J. Wikander, "Holistic Design Optimization in Mechatronics," IFAC Proceedings Volumes, Hangzhou, China, vol. 46(5), pp. 655-662, 2013.

[8] D. Malmquist, J. Wikander, D. Frede D, "Optimal design of harmonic drive servo," IEEE/ASME International Conference on Advanced Intelligent Mechatronics: Mechatronics for Human Wellbeing, Wollongong, Australia, pp.1579-1584, 2013

[9] J. F. Broenink, G. H. Hilderink, J. Eker, L. Sha, "A structured approach to embedded control systems implementation," IEEE International Conference on Control Applications, M. W. Spong, D. Repperger, and J. M. I. Zannatha, Eds. México City, México: IEEE, pp.761-766, 2001.

[10] J. Park and H. Asada, "Concurrent Design Optimization of Mechanical Structure and Control for High Speed Robots," Proc. IEEE Robotics and Automation, San Francisco, CA, USA, 1993.

[11] A. C. Pil and H. Asada (2013), "Integrated structure/control design of mechatronic systems using a recursive experimental optimization method," Mechatronics IEEE/ASME Transactions on Mechatronics, vol. 1(3), pp.191-203, 1996.

[12] M. M. da Silva, O. Brüls, W. Desmet, H. Van Brussela, "Integrated structure and control design for mechatronic systems with configurationdependent dynamics," Mechatronics, vol. 19(6), pp.1016-1025, 2009.
[13] D. Pekarek, A. D. Ames, J. E. Marsden, "Discrete Mechanics and Optimal Control Applied to the Compass Gait Biped," 46th IEEE Conference on Decision and Control, New Orleans, LA, USA, 2007.

[14] S. Samii, A. Cervin, P. Eles, Z. Peng, "Integrated Scheduling and Synthesis of Control Applications on Distributed Embedded Systems," in Proc. Design, Automation and Test in Europe Conference and Exhibition, Nice, France, 2009.

[15] D. Simon, D. Robert, O. Sename, "Control and Real-time Scheduling Co-design Application to Robust Robot Control," 11th IEEE Real Time and Embedded Technology and Applications Symposium, San Francisco, CA. USA, 2005.

[16] D. Simon, A. Seuret, O. Sename, "Real-time control systems : feedback, scheduling and robustness," International Journal of Systems Science, vol. 48(11), pp. 2368-2378, 2017.

[17] A. Cervin, J. Eker, "Control-Scheduling Codesign of Real-Time Systems: The Control Server Approach," Journal of Embedded Computing, 2004.

[18] H. K. Fathy, J. A. Reyer, P. Y. Papalambrous, and A. G. Ulsoy, "On the Coupling between the Plant and Controller Optimization Problems," Proceedings of the American Control Conference, vol. 3, pp.1864-1869, 2001.

[19] F. Roos, H. Johansson, J. Wikander, "Optimal Design of Motor and Gear Ratio in Mechatronic Systems," IFAC Proceedings Volumes, vol. 37(14), pp. 223-228, 2004.

[20] F. Roos, "Towards a methodology for integrated design of mechatronic servo systems," PhD Thesis, KTH, 2007.

[21] D. Malmquist, J. Wikander, "Holistically integrated design of a haptic steering wheel," The 14th Mechatronics Forum International Conference, Karlstad, Sweden, 2014.

[22] J. Awrejcewicz, Z. Koruba, "Classical Mechanics: Applied Mechanics and Mechatronics," Springer-Verlag, Berlin, 250 pp. (ISBN 978-1-46143977-6), 2012.

[23] W. Taha and R. Cartwright, "Some challenges for model-based simulation?," in the 4th Analytic Virtual Integration of Cyber-Physical Systems Workshop, Vancouver, Canada, 2013.

[24] T. Luukkonen, "Modelling and control of quadcopter," aalto uni ersity, Espoo, 9.3,9.4.

[25] M. Mohaqeqi, M. R. Mousavi, and W. Taha, "Conformance testing of cyber-physical systems: A comparative study," in The 14th International Workshop on Automated Verification of Critical Systems, University of Twente, Enschede, Netherlands, 2014.

[26] J. C. Jensen, D. H. Chang, E. A. Lee, "A Model-Based Design Methodology for Cyber-Physical Systems," in Proceedings of The First 
IEEE Workshop on Design, Modeling and Evaluation of Cyber Physical Systems(CyPhy'11), Istanbul, Turkey, 2011. 